\title{
Geo-ecological approach to evaluation of ecological condition of water bodies
}

\author{
Vladimir Smetanin ${ }^{1 *}$, Ilya Tsvetkov ${ }^{1}$ and Andrey Nasonov ${ }^{1}$ \\ ${ }^{1}$ Russian State University of Agriculture, 127550 Timiryazevskaya st., 49, Moscow, Russia
}

\begin{abstract}
Abrstract. The paper proposes a new approach to evaluation of ecological condition of water bodies. The method is based on the use of fractal dimension of the temporal dynamics of water environment pollution components as a quality indicator of state dynamics for a specific type of pollution or an ecosystem in its entirety. The method enables both common integrated and component-based differential evaluation. The writers have identified fractal threshold values that describe the extent of environmental stability of a water system, its resistance to industrial pollution, and probability of further anthropogenic loads. The paper offers a methodology to forecast the ecological condition of water bodies on the basis of quantitative estimation of dynamic fractal parameters representing pollution dynamics of water system components.
\end{abstract}

By definition, an ecosystem is a functional amalgamation of living beings complete with their habitats that persists as long as its self-repairing properties are not compromised. The habitat is directly exposed to anthropogenic impacts that can impair such properties causing reversible or irreversible changes. Therefore, generally an ecosystem should be regarded as a non-equilibrium system of exchange interactions with the environment,where its condition is determined by the degree of its openness (intensity of exchange interactions).

If an ecosystem enters an irreversible state, an environmental disaster ensues; a reversible state causes a crisis. Balance equilibrium exists if the system has not lost its selfrepairing ability. This is the basis of classification of geo-environmental changes in ecosystems triggered by anthropogenic impacts.

Evaluation of ecological condition of water bodies rests on identification of inclusions of negative anthropogenic factors that cause loss of self-repairing properties: the researchers must build a comprehensive description of the water body's functionality and structure, determining the change dynamics of its adaptation resources. The language of fractals is used to describe the ecosystem's change dynamics.

As of now, there is no alternative to the method of evaluating the quality of water bodies other than the "Comprehensive Method of Surface Water Pollution Evaluation by Hydrochemical Parameters" RD 52.24.643-2002, which calculates the unified combinatorial index of water pollution (UCIWP) [1].

The main objective of the UCIWP method is to identify a set of manmade pollutants that persistently impair health and hygiene standards of water quality and consumer

* Corresponding author: smetanin2000@yandex.ru 
standards, and to establish a pollution scale: thus, the method generates a comprehensive functional description of the water body to meet established expert requirements and official quality standards.

The main purpose of the method of Integrated Evaluation of Water Body Ecological Condition (IEWBEC) is to evaluate the ecological condition of the water body by identifying inclusions of adverse anthropogenic factors that cause loss of its self-repairing ability. So, the method not only assesses the quality of aquatic environment, but also the changes dynamics of its adaptive ability through comprehensive description of its functionality and structure, as represented in Table 1.

To describe a water body on the function-logical and logical-physical levels, researchers use a fractal concept according to which complex systems with different scales (hierarchies) exhibit scaling (scale-invariant) properties, and thus the hierarchy, just as geometry of system divisibility, acts as a physical factor that determines the said properties.

Items of technogenesis embedded in the environment represent non-equilibrium flowtype open systems in terms of physics. Flows of energy and matter passing through the systems give rise to self-consistent structures of fractal/scaling type within a broad range of parameters and scales inside the systems, [2].

Table 1. Comprehensive functionality-structure description of a water body that determines the change dynamics of its adapting ability.

\begin{tabular}{|c|c|l|l|}
\hline \multicolumn{2}{|c|}{ Water body description level } & \multicolumn{1}{|c|}{ Water body description character } \\
\hline \multirow{3}{*}{$\begin{array}{c}\text { UCIWP } \\
\text { IEWBE }\end{array}$} & $\begin{array}{c}\text { Functionality } \\
\text { level }\end{array}$ & $\begin{array}{l}\text { Represents the nature of exchange interactions } \\
\text { between the water body and its environment } \\
\text { through time series of control parameters }\end{array}$ \\
\cline { 2 - 4 } & Functionality physics level & $\begin{array}{l}\text { Represents the nature of adaptation processes } \\
\text { through manifestation of scaling (scale-invariant) } \\
\text { system properties. Gives an idea of complementary } \\
\text { behavior of constituent subsystems exposed to } \\
\text { anthropogenic activities. }\end{array}$ \\
\cline { 2 - 4 } & $\begin{array}{l}\text { Represents the comprehensive structure of } \\
\text { adaptation properties of the water body. Gives an } \\
\text { idea of the nature of the water body's adaptive } \\
\text { resource of redistribution responding to changes of } \\
\text { external anthropogenic activities. }\end{array}$ \\
\hline
\end{tabular}

Virtually every natural system, including biological ones, can be described as an open non-equilibrium structure created by a passing flow of matter and energy. Optimal distribution of such factors is ensured by scaling (scale-invariant) structures that act as sort of distributor channels, [3].

Described in terms of mathematics, fractal dimension as a quantitative measure of a structure's scaling disorder connects two types of system characteristics: integral ones that describe the properties of the object in question complete with its local parameters, which emerge after its breakdown. This feature allows representing same-level system descriptions in terms of higher-level models. For example, a water body's differential description on the functional logic level can be easily described to represent its integral properties on the physical logic level.

Thus, fractal description of a water body by its scalability/hierarchy stands alone as a separate coordinate pivotal to register changes in the system's properties at the level of its structure, [4]. 
Changes of fractal dimension values in time illustrate how the system uses its internal resources (natural potential) to adapt when influenced by random changes of external loads.

From this perspective, we can argue that the fractal is a flexible natural mechanism that helps highly involved natural systems with inherent structural heterogeneity achieve most effective distribution of matter and energy under non-equilibrium conditions through changes in their structure.

With every change of the environment's parameters, the system needs to choose the most efficient configuration for its interaction with the environment.

Among transitional form structures, state can be isolated with characteristic values of fractal dimensions $D_{o}, D_{d}$, and $D_{k}$, which were identified as markers that indicate changes of environmental conditions, $[5,6]$.

On this basis, the classification of environmental conditions considers the degree of disorder in the water body structure within the limits of its self-repairing ability. Generalized directed graph (digraph) of conditions shows physical orientation of structural phase transitions respective to identified characteristic fractal dimensions, as seen in Fig. 2.

The above classification suggests that ecological reclamation of a water body (as a set of environmental protection measures) is perceived as the system's forced structural transition from conditions M2 and M3 to conditions M1. The transition is forced by nature because in conditions M3 and M2 the ecosystem loses its self-repairing properties.



Fig. 2. Classification of water body conditions, and generalized digraph of structural phase transitions

The forced transition $M_{3} \Rightarrow \mathrm{M}_{1}$ is generally achieved through reduction of anthropogenic activities in the list of engineering measures that enhance the adaptive capacity of the water body and thus localize the existing excessive anthropogenic load. If the system's resource capabilities to maintain $\mathrm{M}_{1}$ condition are insufficient, the system can spontaneously (inadvertently) revert to its previous stable condition: $M_{1} \Rightarrow \mathrm{M}_{3}$. Forced transitions $M_{2} \Rightarrow \mathrm{M}_{1}$ are achieved by increasing system diversity (more intense interaction 
with the environment); this also contributes to better adaptation ability. Just as in the previous case, spontaneous phase transitions in the other direction $M_{1} \Rightarrow \mathrm{M}_{2}$ also occur.

The spontaneous nature of phase transitions $M_{1} \Rightarrow \mathrm{M}_{3}$ and $M_{1} \Rightarrow \mathrm{M}_{2}$ and the forcible nature of reverse transitions are signes of comparatively low anthropogenic potential of conditions $\mathrm{M}_{3}$ and $\mathrm{M}_{2}$ compared to conditions $\mathrm{M}_{1}$ that are more complex in terms the degrees of freedom and ensure optimized load distribution thanks to structure scaling.

Therefore, the use of fractal dimension in the classification registers both stable environmental conditions and their intermediate forms, which is impossible for conventional topological dimensioning (UCIWP). When the latter is used as a quantitative evaluation unit for conditions, this invariably results in loss of significant solutions.

In the particular case, characteristic fractal dimensions are found individually for each dynamic system with multifractal dynamics; however, studies have demonstrated that their size is rather common for complex dynamic systems of vastly different types (natural, biological, socioeconomic), [5].

See Table 2 for characteristic values of fractal dimensions of a water body ecosystem, and specifics of its dynamics.

Table 2. Characteristic values of fractal dimensions of a water body ecosystem, and specifics of its dynamics

\begin{tabular}{|c|c|c|}
\hline $\begin{array}{c}\text { Fractal } \\
\text { dimension }\end{array}$ & Character of ecological condition & Specifics of system dynamics \\
\hline $\begin{array}{c}D_{d}=1- \\
1.2\end{array}$ & $\begin{array}{c}\text { Human-dominated state, causing loss of } \\
\text { self-repairing properties, stagnation and } \\
\text { lack of inherent growth }\end{array}$ & $\begin{array}{c}\text { System behavior is strictly regulated } \\
\text { by unidirectional dominant factors of } \\
\text { anthropogenic influences }\end{array}$ \\
\hline $\begin{array}{c}D_{0}= \\
1.4 \pm 0.15\end{array}$ & $\begin{array}{c}\text { Optimally balanced condition with the } \\
\text { environment, no loss of self-repairing } \\
\text { properties. }\end{array}$ & $\begin{array}{c}\text { Steady and stable dynamics with } \\
\text { gradual variations of system } \\
\text { characteristics over a fairly narrow } \\
\text { range }\end{array}$ \\
\hline $\begin{array}{c}D_{k}= \\
1.7 \pm 0.15\end{array}$ & $\begin{array}{c}\text { Unstable state of crisis, loss of self- } \\
\text { repairing properties, reduced diversity in } \\
\text { the original ecosystem. }\end{array}$ & $\begin{array}{c}\text { Unstable ecosystem dynamics, with } \\
\text { abrupt leaps of system characteristics }\end{array}$ \\
\hline
\end{tabular}

In terms of mathematics, each subset of classified conditions $M_{s}=\left\langle\mathrm{M}_{1} \cup \mathrm{M}_{2} \cup_{3} \mathrm{UM}_{4}\right\rangle$ possesses isomorphism - a similar structure within a limited range of fractal dimension values that indicate persistence of specific properties/attributes of a water body interacting with the environment:

1. Optimality of environment: fractal dimensions of the water body focus in the interval $\left(D_{d}-D_{o}\right)-$ a sign of self-regulating conditions where the environment structure corresponds to intensity of anthropogenic impact;

2. Criticality of environment: fractal dimensions of the water body focus in the interval $\left(D_{k}\right.$ $-D_{o}$ ) - a sign of severe manifest instability of conditions, while diversity in the original water body (partial "burning" of the environment) is reduced to compensate for preserved stability against excessive activity of human impact;

3. Stagnation of environment: fractal dimension of the water body focus in the interval ( 1 $-D_{d}$ ) - a sign of stagnation and lack of evolution in the ecosystem, as degeneration with manifest anthropogenic domination; 
4. Ecological disaster: fractal dimension of the water body focus in the interval $\left(D_{k}-2\right)-$ a sign of uncontrollable destruction/collapse of the water body ecosystem and transition to other qualitatively different ecological forms of existence.

As an analytical form is a complex description of the water body, we propose a ratio in which the entire array of ecological conditions $M_{s}=\left\langle\mathrm{M}_{1} \mathrm{UM}_{2} \mathrm{UM}_{3} \mathrm{UM}_{4}\right\rangle$ is described with a digraph that determines physical orientation of structural phase transitions:

$$
M_{s}=\left\langle A, \mathrm{~F}, \mathrm{~S}, I^{*}, \Pi\left(\mathrm{F}, \mathrm{S}, \mathrm{I}^{*}\right)\right\rangle
$$

Where $\mathrm{A}$ is the object structure of a complex-structure system; F- submodel that presents the system's material properties or functions, showing the nature of exchange interaction with the environment over a certain period of observation; S- submodel to describe the structure of adaptation processes through manifestations of the system's scaling (scale-invariant) properties;

$\mathrm{I}^{*}$ - integrated structure of the water body in an active environment; $\Pi_{\mathrm{f}}\left(\mathrm{SI}^{*}\right)-$ limits to functional integrity of the water body.

Formally, the ratios are determined by the terms of $\mathrm{F}$ imaged against $\mathrm{S}$ to ensure integral distribution of adaptation potential $\Pi_{\mathrm{f}}\left(\mathrm{I}^{*}\right)$ relative to critical signs of fractal system violations.

In digraph (1), submodel (F) that represents essential properties or functions of the system that represents the nature of exchange interactions with the environment for a specific period of observation, is represented with time series of the anthropogenic load parameters in question, which are complex curves of irregular interaction between the water body and the environment. The structure of parameters is regulated by GOST 17.1.307-82 "Rules of Quality Control for Water Reservoirs and Ducts".

The submodel (S) that describes the structure of adaptation processes through manifestations of the system's scaling (scale-invariant) properties by its scalability coordinate, is expressed analytically by measuring the lengths of time series curves invariant to rescaling (fractal curves). A fractal curve in time interval $t \in[a, b]$ is defined as a continuous and differentiable curve, the length of which depends on the averaging scale, [7].

Fractal feature of the water body generates structural trends (gradients) of its adaptability, expressed numerically through fractal dimension.

Integrated structure of the water body in an active environment ( $\left.I^{*}\right)$ is graphically expressed through the multifractal phase portrait: a geometric image of the water body in the point of monitoring.

Limits to functional integrity of the water body $\left[\Pi_{\phi}\left(\mathrm{SI}^{*}\right)\right]$ are set by the limits of selfrecovery of its structure, and expressed through system coefficients of differential and integral stability.

To calculate coefficients of differential stability, the following ratios are used.

Differential rates of inertia

$$
\begin{array}{r}
\Delta K_{d}=K_{d}\left(D_{i}^{t+1}\right)-K_{d}\left(D_{i}^{t}\right) \\
K_{d}\left(D_{i}^{t}\right)=D_{i}^{t}-D_{d} \\
K_{d}\left(D_{i}^{t+1}\right)=D_{i}^{t+1}-D_{d}
\end{array}
$$

Where $\Delta K_{d}$ - differential coefficient of inertia hydrochemical indices; $K_{d}\left(D_{i}^{t}\right)$; $K_{d}\left(D_{i}^{t+1}\right)$ - differential coefficients of stability of hydrochemical indicators for successive 
time intervals $\mathrm{t}$ and $(\mathrm{t}+1)$ respectively; $D_{d}$ - fractal indicator of inertia (anthropogenic domination)

Differential rates of optimality:

$$
\begin{gathered}
\Delta K_{0}=K_{0}\left(D_{i}^{t+1}\right)-K_{0}\left(D_{i}^{t}\right) \\
K_{0}\left(D_{i}^{t}\right)=D_{i}^{t}-D_{0} \\
K_{0}\left(D_{i}^{t+1}\right)=D_{i}^{t+1}-D_{0}
\end{gathered}
$$

Where $\Delta K_{0}$ - differential coefficient of optimal hydrochemical indicators; $D_{0}$ - fractal indicator of balance stability

Differential rates of criticality:

$$
\begin{gathered}
\Delta K_{k}=K_{k}\left(D_{i}^{t+1}\right)-K_{k}\left(D_{i}^{t}\right) \\
K_{k}\left(D_{i}^{t}\right)=D_{i}^{t}-D_{k} \\
K_{k}\left(D_{i}^{t+1}\right)=D_{i}^{t+1}-D_{k}
\end{gathered}
$$

Where $\Delta K_{k}$ - differential coefficient of critical hydro-chemical indicators; $\mathrm{D}_{\mathrm{k}}$ - fractal indicator of anthropogenic criticality.

To calculate integral coefficient of stability, we use the following ratios.

Integral coefficients of inertia:

$$
\begin{gathered}
\Delta K_{d}^{n}=K_{d}^{n}\left(D_{i}^{t+1}\right)-K_{d}^{n}\left(D_{i}^{t}\right) \\
K_{d}^{n}=\frac{\sum_{1}^{n}\left(D_{i}^{t}-D_{d}\right)}{n} \\
K_{d}^{n}\left(D_{i}^{t+1}\right)=\frac{\sum_{1}^{n}\left(D_{i}^{t+1}-D_{d}\right)}{n}
\end{gathered}
$$

Where $\Delta K_{d}^{n}$ - integral coefficients of inertia by hydrochemical indicators; $K_{d}^{n}\left(D_{i}^{t}\right)$, $K_{d}^{n}\left(D_{i}^{t+1}\right)$ - integral differential coefficients of inertia for successive points in time $\mathrm{t}$ and $(\mathrm{t}+1)$ respectively; $n-$ number of factors (control components).

Integral coefficients of optimality:

$$
\begin{gathered}
\Delta K_{0}^{n}=K_{0}^{n}\left(D_{i}^{t+1}\right)-K_{0}^{n}\left(D_{i}^{t}\right) \\
K_{0}^{n}\left(D_{i}^{t}\right)=\frac{\sum_{1}^{n}\left(D_{i}^{t}-D_{0}\right)}{n} \\
K_{0}^{n}\left(D_{i}^{t+1}\right)=\frac{\sum_{1}^{n}\left(D_{i}^{\mathrm{e}+1}-D_{0}\right)}{n},
\end{gathered}
$$

Where $\Delta K_{0}^{n}$ - integral coefficients of optimality by hydrochemical indicators; $K_{0}^{n}\left(D_{i}^{t}\right)$, $K_{0}^{n}\left(D_{i}^{t+1}\right)$ - integral differential coefficients of stability for successive points in time $\mathrm{t}$ and $(\mathrm{t}+1)$ respectively.

Integral coefficients of criticality: 


$$
\begin{gathered}
\Delta K_{k}^{n}=K_{k}^{n}\left(D_{i}^{t+1}\right)-K_{k}^{n}\left(D_{i}^{t}\right) \\
K_{k}^{n}\left(D_{i}^{t}\right)=\frac{\sum_{1}^{n}\left(D_{i}^{t}-D_{k}\right)}{n} \\
K_{k}^{n}\left(D_{i}^{t+1}\right)=\frac{\sum_{1}^{n}\left(D_{i}^{\mathrm{e}+1}-D_{k}\right)}{n},
\end{gathered}
$$

Where $\Delta K_{k}^{n}$ - integral coefficients of criticality by hydrochemical indicators; $K_{k}^{n}\left(D_{i}^{t}\right)$, $K_{k}^{n}\left(D_{i}^{t+1}\right)$-integral differential coefficients of criticality for successive points in time $\mathrm{t}$ and $(\mathrm{t}+1)$ respectively.

Limits to structural stability $\Pi_{\mathrm{f}}\left(\mathrm{F}, \mathrm{S}, \mathrm{I}^{*}\right)$ are used to evaluate the resulting condition of the water body and the underlying anthropogenic load parameters.

In particular, the resulting ecological condition of a water body is evaluated by integral coefficients of stable condition through the following formulas:



$$
\begin{aligned}
& K_{k}^{n}\left(D_{k}^{t}\right) \quad K_{k}^{n}\left(D_{i}^{t+1}\right) \quad \Delta K_{k}^{n}
\end{aligned}
$$

The physical sense of mathematical condition (8) is that the system remains in the subset of conditions $M_{s}=\left\langle\mathrm{M}_{1} ; \mathrm{M}_{2} ; \mathrm{M}_{3} ; \mathrm{M}_{4}\right\rangle$ [9], in which the system's adaptation potential is used in the most efficient way; this determined by proximity of the integral coefficient to one of such subsets that formally determine similarity of structural parameters (and therefore of the system's manifest properties). The sign of the differential coefficient points to either positive or negative dynamic that determines stability of the system's condition/properties [10].

The anthropogenic risk factors are measured by differential coefficients of stability to meet the condition:

$$
\mathrm{F}_{\mathrm{r}}=\min \left\langle\Pi_{\mathrm{f}}(S)\right\rangle ;\left\langle\Pi_{\mathrm{f}}(S)\right\rangle=\begin{aligned}
& K_{d}\left(D_{i}^{t}\right) ; K_{d}\left(D_{i}^{t+1}\right) ; \Delta K_{d} \\
& K_{k}\left(D_{i}^{t}\right) ; K_{k}\left(D_{i}^{t+1}\right) ; \Delta K_{k}
\end{aligned}
$$

The physical sense of mathematical condition (9) is that stability of the selected condition can be compromised by specific anthropogenic risk factors, each of which is determined by proximity of their differential coefficients to their critical values.

Existence of anthropogenic risk in the factor system can result in energy-inefficient integral redistribution of its adaptation potential, thus causing undesirable simplification of the system: the environment "burns out" or loses its ecological diversity.

The extreme of such simplification is when the ecosystem loses its fractal properties this means transition from the fractal to the uniform mode, [8].

An integrated model formally describes the water body as an ecosystem with a nonuniform and non-equilibrium structure. The model's fractal component helps to register differences in the water body's adaptive responses over the range of hydrochemical parameters, thus helping to measure the development level of its structure. The more developed is the water body structure (hierarchical micro-levels of the system), the more manifest is its self-consistent behavior with respect to critical fluctuations of the environment: the stronger are the system's scaling/fractal properties S. 
This is submodel that describes system by its scaling coordinate. It is analytically expressed by measured lengths of time series curves that are rescaling-invariant (fractal) curves.

In the time interval $t \in[a, b]$, a fractal curve is defined as a continuous undifferentiated curve of which the length depends on the averaging scale, [7]. Fractal property of the water body generates structural trends (gradients) of its adaptability, expressed numerically with fractal dimension.

In this way, one generates a set of differential descriptions of adaptive response systems and their limitations $<\mathrm{S} ; \Pi_{\mathrm{f}}\left(\mathrm{S}^{*}\right)>$ on the impact of respective pollution parameters.

Integral properties of the water body and their limits $\left\langle\mathrm{I}^{*} ; \Pi_{\mathrm{f}}\left(\mathrm{I}^{*}\right)\right\rangle$ are described on the logical-physical level through a multifractal phase image that clearly represents the dynamics of redistribution of adaptation potential responding to changes of interaction with the environment.

Structural limits $\Pi_{\mathrm{f}}\left(\mathrm{F}, \mathrm{S}, \mathrm{I}^{*}\right)$ [11] help to quantify redistribution of the water body's adaptation resource (natural potential) responding to changes of anthropogenic activities, and to see how this redistribution brings the system closer to adverse environmental conditions or on the contrary maintains optimized balanced mode of interaction with the environment.

In this way, the method comprehensively evaluates the water body's ecological condition and highlights the risk factors that jeopardize it with their positive trend. Quantification of such factors helps to assess their critical impact on the system's structural balance.

Fractal description of the water body generates uniform descriptions of various processes of interaction with the environment that are different by their physical nature. The method can be used successfully to create descriptions of both biotic and hydrochemical parameters of the ecosystem; the descriptions can be later correlated.

The model clearly visualizes the dynamics of inclusion of new or exclusion of old factors of anthropogenic impact on the water body, and then can assess criticality of these inclusions. This feature of the integrated model is useful in natural environment management, planning and examination of efficiency of rehabilitation activities.

To match the integrated model (1), we propose the following generalized algorithm for Integrated Evaluation of Water Body Ecological Condition (IEWBEC) - see Figure 3. 


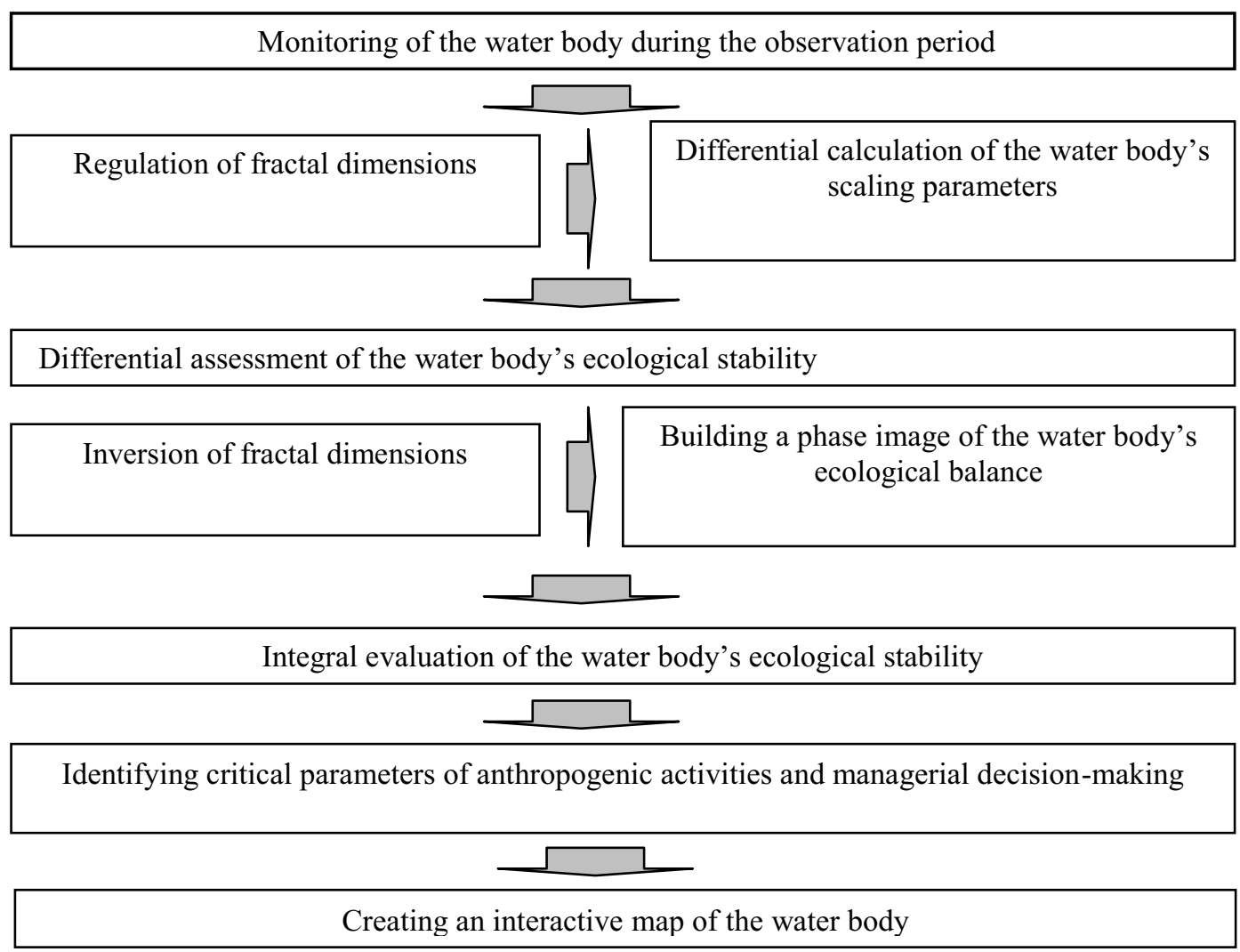

Fig. 3. Generalized algorithm of IEWBEC

\section{References}

1. RD 52.24.643-2002, Rosgidromet (2003)

2. D.I. Iudin, Bulletin of Samara Scientific Center under Russian Academy of Sciences 7, 248-251 (2005)

3. V.N. Yakimov, L.A. Solncev, G.S. Rosenberg, D.I. Iudin, D.B. Gelashvili, Ontogenes 45, 207-216 (2014)

4. A.D. Titov, F.I. Mavrikidi, Korolev SGAU 1,127 (2011)

5. A.N. Kudinov, V.P. Tsvetkov, I.V. Tsvetkov, Russian Journal of Mathematical Physics 18, 149-155 (2011)

6. A.N. Nasonov, I.V. Tsvetkov, A.V. Nikiforov, Prirodoobustroystvo Publishers 1, 69-78 (2016)

7. A.N. Kudinov, O.I. Krylova, V.P. Tsvetkov, I.V. Tsvetkov, Mathematical Journal 5, 5260 (2014)

8. D.I. Iudin, A.V. Chechin, O.V. Kaschenko, Information and Cosmos Publishers 1, 8487 (2014)

9. V.I. Smetanin, A.N. Nasonov, I.V. Tsvetkov, Nelineyniy Mir 5, 67-69 (2015)

10. A.N. Kudinov, D.Y. Lebedev, V.P. Tsvetkov, I.V. Tsvetkov, Mathematical Models and Computer Simulations 3 (2015)

11. I.V. Tsvetkov, O.I. Krilova, Software and Systems Publishers 4 (2015) 
12. A.P. Ivanov, A.N. Kudinov, D.Y. Lebedev, Mathematical Models and Computer Simulations 1 (2016)

13. A.N. Kudinov, S.A. Mikheyev, I.V. Tsvetkov, Inseries: Applied Mathematics 1 (2014)

14. V.I. Smetanin, A.N. Nasonov, I.M. Zhogin, I.V. Tsvetkov, Prirodoobustroystvo Publishers 5 (2013) 